\title{
Convergence Rates for the Iteratively Regularized Landweber Iteration in Banach Space
}

\author{
Barbara Kaltenbacher* \\ University of Klagenfurt, \\ Universitätsstraße 65-67, 9020 Klagenfurt, Austria \\ barbara.kaltenbacher@aau.at
}

\begin{abstract}
In this paper we provide a convergence rates result for a modified version of Landweber iteration with a priori regularization parameter choice in a Banach space setting.
\end{abstract}

Keywords: regularization, nonlinear inverse problems, Banach space, Landweber iteration.

An increasing number of inverse problems is nowadays posed in a Banach space rather than a Hilbert space setting, cf., e.g., [2613] and the references therein.

An Example of a model problem, where the use of non-Hilbert Banach spaces is useful, is the identification of the space-dependent coefficient function $c$ in the elliptic boundary value problem

$$
\begin{array}{rlrl}
-\Delta u+c u & =f & & \text { in } \Omega \\
u=0 & & \text { on } \partial \Omega
\end{array}
$$

from measurements of $u$ in $\Omega \subseteq \mathbb{R}^{d}, d \in\{1,2,3\}$, where $f$ is assumed to be known. Here e.g., the choices $p=1$ for recovering sparse solutions, $q=\infty$ for modelling uniformly bounded noise, or $q=1$ for dealing with impulsive noise are particulary promising, see, e.g., 3] and the numerical experiments in Section 7.3.3 of 13 .

Motivated by this fact we consider nonlinear ill-posed operator equations

$$
F(x)=y
$$

where $F$ maps between Banach spaces $X$ and $Y$.

In the example above, the forward operator $F$ maps the coefficient function $c$ to the solution of the boundary value problem (10), (2), and is well-defined as an operator

$$
F: \mathcal{D}(F) \subseteq L^{p}(\Omega) \rightarrow L^{q}(\Omega),
$$

* Support by the German Science Foundation DFG under grant KA 1778/5-1 and within the Cluster of Excellence in Simulation Technology (EXC 310/1) at the University of Stuttgart is gratefully acknowledged.

D. Hömberg and F. Tröltzsch (Eds.): CSMO 2011, IFIP AICT 391, pp. 38 48, 2013.

(C) IFIP International Federation for Information Processing 2013 
where $\mathcal{D}(F)=\left\{c \in X \mid \exists \hat{c} \in L^{\infty}(\Omega), \hat{c} \geq 0\right.$ a.e. : $\left.\|c-\hat{c}\|_{X} \leq r\right\}, r$ sufficiently small, for any

$$
\begin{array}{ll}
p, q \in[1, \infty], \quad f \in L^{1}(\Omega) & \text { if } d \in\{1,2\} \\
p \in[1, \infty], q \in\left(\frac{d}{2}, \infty\right], f \in L^{s}(\Omega), s>\frac{d}{2} & \text { if } d \geq 3,
\end{array}
$$

see Section 1.3 in 13 .

Since the given data $y^{\delta}$ are typically contaminated by noise, regularization has to be applied. We are going to assume that the noise level $\delta$ in

$$
\left\|y-y^{\delta}\right\| \leq \delta
$$

is known and provide convergence results in the sense of regularization methods, i.e., as $\delta$ tends to zero. In the following, $x_{0}$ is some initial guess and we will assume that a solution $x^{\dagger}$ to (3) exists.

Variational methods in Banach space have been extensively studied in the literature, see, e.g., 2010] and the references therein.

Since these generalizations of Tikhonov regularization require computation of a global minimizer, iterative methods are an attractive alternative especially for large scale problems. After convergence results on iterative methods for nonlinear ill-posed operator equations in Banach spaces had already been obtained in the 1990's (cf. the references in [1]) in the special case $X=Y$, the general case $X \neq Y$ has only been treaten quite recently, see e.g. [5], 7], and [9] for an analysis of gradient and Newton type iterations. While convergence rates have already been established for the iteratively regularized Gauss-Newton iteration in [7, the question of convergence rates remains challenging and will be tackled in this paper; we refer to 14 for a different approach.

In order to formulate and later on analyze the method, we have to introduce some basic notations and concepts.

Consider, for some $q \in(1, \infty)$, the duality mapping $J_{q}^{X}(x):=\partial\left\{\frac{1}{q}\|x\|^{q}\right\}$, which maps from $X$ to its dual $X^{*}$. To analyze convergence rates we employ the Bregman distance

$$
D_{j_{q}}(\tilde{x}, x)=\frac{1}{q}\|\tilde{x}\|^{q}-\frac{1}{q}\|x\|^{q}-\left\langle j_{q}^{X}(x), \tilde{x}-x\right\rangle_{X^{*} . X}
$$

(where $j_{q}^{X}(x)$ denotes a single valued selection of $J_{q}^{X}(x)$ ) or its shifted version

$$
D_{q}^{x_{0}}(\tilde{x}, x):=D_{j_{q}}\left(\tilde{x}-x_{0}, x-x_{0}\right) .
$$

Throughout this paper we will assume that $X$ is smooth, which means that the duality mapping is single-valued, and moreover, that $X$ is $q$-convex, i.e.,

$$
D_{j_{q}}(x, y) \geq c_{q}\|x-y\|^{q}
$$

for some constant $c_{q}>0$. As a consequence, $X$ is reflexive and we also have

$$
D_{j_{q^{*}}}\left(x^{*}, y^{*}\right) \leq C_{q^{*}}\left\|x^{*}-y^{*}\right\|^{q^{*}},
$$


for some $C_{q^{*}}$. Here $q^{*}$ denotes the dual index $q^{*}=\frac{q}{q-1}$. Moreover, the duality mapping is bijective and $J_{q}^{-1}=J_{q^{*}}^{X^{*}}$, the latter denoting the (by $q$-convexity also single-valued) duality mapping on $X^{*}$. We will also make use of the identities

$$
D_{j_{q}}(x, y)=D_{j_{q}}(x, z)+D_{j_{q}}(z, y)+\left\langle J_{q}^{X}(z)-J_{q}^{X}(y), x-z\right\rangle_{X^{*}, X}
$$

and

$$
D_{j_{q}}(y, x)=D_{j_{q^{*}}}\left(J_{q}^{X}(x), J_{q}^{X}(y)\right) .
$$

For more details on the geometry of Banach spaces we refer, e.g., to [12] and the references therein.

We here consider the iteratively regularized Landweber iteration

$$
\begin{aligned}
J_{q}^{X}\left(x_{n+1}^{\delta}-x_{0}\right) & =\left(1-\alpha_{n}\right) J_{q}^{X}\left(x_{n}^{\delta}-x_{0}\right)-\mu_{n} A_{n}^{*} j_{p}^{Y}\left(F\left(x_{n}^{\delta}\right)-y^{\delta}\right), \\
x_{n+1}^{\delta} & =x_{0}+J_{q^{*}}^{X^{*}}\left(J_{q}^{X}\left(x_{n+1}^{\delta}-x_{0}\right)\right), \quad n=0,1, \ldots
\end{aligned}
$$

where we abbreviate

$$
A_{n}=F^{\prime}\left(x_{n}^{\delta}\right),
$$

which, for an appropriate choice of the sequence $\left\{\alpha_{n}\right\}_{n \in \mathbb{N}} \in(0,1]$, has been shown to be convergent with rates under a source condition

$$
\left.x^{\dagger}-x_{0} \in \mathcal{R}\left(F^{\prime}\left(x^{\dagger}\right)^{*} F^{\prime}\left(x^{\dagger}\right)\right)^{\nu / 2}\right),
$$

with $\nu=1$ in a Hilbert space setting in [11. Since the linearized forward operator $F^{\prime}(x)$ typically has some smoothing property (reflecting the ill-posedness of the inverse problems) condition (10) can often be interpreted as a regularity assumption on the initial error $x^{\dagger}-x_{0}$, which is stronger for larger $\nu$.

In the Hilbert space case the proof of convergence rates for the plain Landweber iteration (i.e., (9) with $\alpha_{n}=0$ ) under source conditions (10) relies on the fact that the iteration errors $x_{n}^{\delta}-x^{\dagger}$ remain in the range of $\left(F^{\prime}\left(x^{\dagger}\right)^{*} F^{\prime}\left(x^{\dagger}\right)\right)^{\nu / 2}$ and their preimages under $\left(F^{\prime}\left(x^{\dagger}\right)^{*} F^{\prime}\left(x^{\dagger}\right)\right)^{\nu / 2}$ form a bounded sequence (cf., Proposition 2.11 in [8]). Since carrying over this approach to the Banach space setting would require more restrictive assumptions on the structure of the spaces even in the special case $\nu=1$, we here consider the modified version with an appropriate choice of $\left\{\alpha_{n}\right\}_{n \in \mathbb{N}} \in(0,1]$.

In place of the Hilbert space source condition (10), we consider variational inequalities

$$
\begin{aligned}
& \exists \beta>0 \forall x \in \mathcal{B}_{\rho}^{D}\left(x^{\dagger}\right): \\
& \left|\left\langle J_{q}^{X}\left(x^{\dagger}-x_{0}\right), x-x^{\dagger}\right\rangle_{X^{*} \times X}\right| \leq \beta D_{q}^{x_{0}}\left(x^{\dagger}, x\right)^{\frac{1-\nu}{2}}\left\|F^{\prime}\left(x^{\dagger}\right)\left(x-x^{\dagger}\right)\right\|^{\nu},
\end{aligned}
$$

cf., e.g., 4], where

$$
\mathcal{B}_{\rho}^{D}\left(x^{\dagger}\right)=\left\{x \in X \mid D_{q}^{x_{0}}\left(x^{\dagger}, x\right) \leq \rho^{2}\right\}
$$

with $\rho>0$ such that $x_{0} \in \mathcal{B}_{\rho}^{D}\left(x^{\dagger}\right)$. Using the interpolation and the CauchySchwarz inequality, it is readily checked that in the Hilbert space case (10) 
implies (11). For more details on such variational inequalities we refer to Section 3.2 .3 in 13 and the references therein.

The assumptions on the forward operator besides a condition on the domain

$$
\mathcal{B}_{\rho}^{D}\left(x^{\dagger}\right) \subseteq \mathcal{D}(F)
$$

include a structural condition on its degree of nonlinearity (cf. [4]

$$
\begin{aligned}
& \left\|\left(F^{\prime}\left(x^{\dagger}+v\right)-F^{\prime}\left(x^{\dagger}\right)\right) v\right\| \leq K\left\|F^{\prime}\left(x^{\dagger}\right) v\right\|^{c_{1}} D_{q}^{x_{0}}\left(x^{\dagger}, v+x^{\dagger}\right)^{c_{2}}, \\
& v \in X, x^{\dagger}+v \in \mathcal{B}_{\rho}^{D}\left(x^{\dagger}\right)
\end{aligned}
$$

whose strength depends on the smoothness index in (11). Namely, we assume that

$$
\begin{gathered}
c_{1}=1 \text { or } c_{1}+c_{2} p>1 \text { or }\left(c_{1}+c_{2} p \geq 1 \text { and } K \text { is sufficiently small }\right) \\
c_{1}+c_{2} \frac{2 \nu}{\nu+1} \geq 1,
\end{gathered}
$$

so that in case $\nu=1$, a Lipschitz condition on $F^{\prime}$, corresponding to $\left(c_{1}, c_{2}\right)=$ $(0,1)$ is sufficient.

Here $F^{\prime}$ denotes the Gateaux derivative of $F$, hence a Taylor remainder estimate

$$
\begin{aligned}
& \left\|F\left(x_{n}^{\delta}\right)-F\left(x^{\dagger}\right)-F^{\prime}\left(x^{\dagger}\right)\left(x_{n}^{\delta}-x^{\dagger}\right)\right\| \\
& =\left\|g(1)-g(0)-F^{\prime}\left(x^{\dagger}\right)\left(x_{n}^{\delta}-x^{\dagger}\right)\right\| \\
& =\left\|\int_{0}^{1} g^{\prime}(t) d t-F^{\prime}\left(x^{\dagger}\right)\left(x_{n}^{\delta}-x^{\dagger}\right)\right\| \\
& =\left\|\int_{0}^{1} F^{\prime}\left(x^{\dagger}+t\left(x_{n}^{\delta}-x^{\dagger}\right)\right)\left(x_{n}^{\delta}-x^{\dagger}\right) d t-F^{\prime}\left(x^{\dagger}\right)\left(x_{n}^{\delta}-x^{\dagger}\right)\right\| \\
& \leq K\left\|F^{\prime}\left(x^{\dagger}\right)\left(x_{n}^{\delta}-x^{\dagger}\right)\right\|^{c_{1}} D_{q}^{x_{0}}\left(x^{\dagger}, x_{n}^{\delta}\right)^{c_{2}}
\end{aligned}
$$

where $g: t \mapsto F\left(x^{\dagger}+t\left(x_{n}^{\delta}-x^{\dagger}\right)\right)$, follows from (13).

We will assume that in each step the step size $\mu_{n}>0$ in (9) is chosen such that

$$
\mu_{n} \frac{1-3 C\left(c_{1}\right) K}{3\left(1-C\left(c_{1}\right) K\right)}\left\|F\left(x_{n}^{\delta}\right)-y^{\delta}\right\|^{p}-2^{q^{*}+q-2} C_{q^{*}} \mu_{n}^{q^{*}}\left\|A_{n}^{*} j_{p}^{Y}\left(F\left(x_{n}^{\delta}\right)-y^{\delta}\right)\right\|^{q^{*}} \geq 0
$$

where $C\left(c_{1}\right)=c_{1}^{c_{1}}\left(1-c_{1}\right)^{1-c_{1}}$, and $c_{1}, K$ are as in (13), which is possible, e.g., by a choice $0<\mu_{n} \leq C_{\mu} \frac{\left\|F\left(x_{n}^{\delta}\right)-y^{\delta}\right\|^{\frac{q-p}{q-1}}}{\left\|A_{n}\right\|^{q^{*}}}=: \bar{\mu}_{n}$ with $C_{\mu}:=\frac{2^{2-q^{*}-q}}{3} \frac{1-3 C\left(c_{1}\right) K}{\left(1-C\left(c_{1}\right) K\right) C_{q^{*}}}$ If

$$
p \geq q
$$

and $F, F^{\prime}$ are bounded on $\mathcal{B}_{\rho}^{D}\left(x^{\dagger}\right)$, it is possible to bound $\bar{\mu}_{n}$ away from zero

$$
\bar{\mu}_{n} \geq C_{\mu}\left(\sup _{x \in \mathcal{B}_{\rho}^{D}\left(x^{\dagger}\right)}(\|F(x)-y\|+\bar{\delta})^{p-q}\left\|F^{\prime}(x)\right\|^{q}\right)^{-1 /(q-1)}=: \underline{\mu}
$$


for $\delta \in[0, \bar{\delta}]$, provided the iterates remain in $\mathcal{B}_{\rho}^{D}\left(x^{\dagger}\right)$ (which we will show by induction in the proof of Theorem 1). Hence, there exist $\underline{\mu}, \bar{\mu}>0$ independent of $n$ and $\delta$ such that we can choose

$$
0<\underline{\mu} \leq \mu_{n} \leq \bar{\mu}
$$

(e.g., by simply setting $\mu_{n} \equiv \underline{\mu}$ ).

Moreover, we will use an a priori choice of the stopping index $n_{*}$ according to

$$
n_{*}(\delta)=\min \left\{n \in \mathbb{N}: \alpha_{n}^{\frac{\nu+1}{p(\nu+1)-2 \nu}} \leq \tau \delta\right\}
$$

and of $\left\{\alpha_{n}\right\}_{n \in \mathbb{N}}$ such that

$$
\left(\frac{\alpha_{n+1}}{\alpha_{n}}\right)^{\frac{2 \nu}{p(\nu+1)-2 \nu}}+\frac{1}{3} \alpha_{n}-1 \geq c \alpha_{n}
$$

for some $c \in\left(0, \frac{1}{3}\right)$ independent of $n$, where $\nu \in[0,1]$ is the exponent in the variational inequality (11).

Remark 1. A possible choice of $\left\{\alpha_{n}\right\}_{n \in \mathbb{N}}$ satisfying (23) and smallness of $\alpha_{\max }$ is given by

$$
\alpha_{n}=\frac{\alpha_{0}}{(n+1)^{x}}
$$

with $x \in(0,1]$ such that $3 x \theta<\alpha_{0}$ sufficiently small, since then with $c:=$ $\frac{1}{3}-\frac{x \theta}{\alpha_{0}}>0$, using the abbreviation $\theta=\frac{2 \nu}{p(\nu+1)-2 \nu} \in\left[0, \frac{1}{p-1}\right]$ we get by the Mean Value Theorem

$$
\begin{aligned}
& \left(\frac{\alpha_{n+1}}{\alpha_{n}}\right)^{\theta}+\left(\frac{1}{3}-c\right) \alpha_{n}-1 \\
& =\frac{\alpha_{n}}{\alpha_{0}}\left\{\alpha_{0}\left(\frac{1}{3}-c\right)-\frac{(n+2)^{x \theta}-(n+1)^{x \theta}}{(n+2)^{x \theta}}(n+1)^{x}\right\} \\
& =\frac{\alpha_{n}}{\alpha_{0}}\left\{\alpha_{0}\left(\frac{1}{3}-c\right)-\frac{x \theta(n+1+t)^{x \theta-1}}{(n+2)^{x \theta}}(n+1)^{x}\right\} \\
& \geq \frac{\alpha_{n}}{\alpha_{0}}\left\{\alpha_{0}\left(\frac{1}{3}-c\right)-x \theta \frac{(n+1)^{x}}{n+1+t}\right\} \geq 0,
\end{aligned}
$$

for some $t \in[0,1]$.

Theorem 1. Assume that $X$ is smooth and $q$-convex, that $x_{0}$ is sufficiently close to $x^{\dagger}$, i.e., $x_{0} \in \mathcal{B}_{\rho}^{D}\left(x^{\dagger}\right)$, (which by (5) implies that $\left\|x^{\dagger}-x_{0}\right\|$ is also small), that a variational inequality (11) with $\nu \in(0,1]$ and $\beta$ sufficiently small is satisfied, that $F$ satisfies (13) with (14), (15), that $F$ and $F^{\prime}$ are continuous and uniformly bounded in $\mathcal{B}_{\rho}^{D}\left(x^{\dagger}\right)$, that $(12)$ holds and that

$$
q^{*} \geq \frac{2 \nu}{p(\nu+1)-2 \nu}+1 .
$$


Let $n_{*}(\delta)$ be chosen according to (22) with $\tau$ sufficiently large. Moreover assume that (19) holds and the sequence $\left\{\mu_{n}\right\}_{n \in \mathbb{N}}$ is chosen such that (21) holds for $0<\underline{\mu}<\bar{\mu}$ according to (20), and let the sequence $\left\{\alpha_{n}\right\}_{n \in \mathbb{N}} \subseteq[0,1]$ be chosen such that (23) holds, and $\alpha_{\max }=\max _{n \in \mathbb{N}} \alpha_{n}$ is sufficiently small.

Then, the iterates $x_{n+1}^{\delta}$ remain in $\mathcal{B}_{\rho}^{D}\left(x^{\dagger}\right)$ for all $n \leq n_{*}(\delta)-1$ with $n_{*}$ according to (22). Moreover, we obtain optimal convergence rates

$$
D_{q}^{x_{0}}\left(x^{\dagger}, x_{n_{*}}\right)=O\left(\delta^{\frac{2 \nu}{\nu+1}}\right), \quad \text { as } \delta \rightarrow 0
$$

as well as in the noise free case $\delta=0$

$$
D_{q}^{x_{0}}\left(x^{\dagger}, x_{n}\right)=O\left(\alpha_{n}^{\frac{2 \nu}{p(\nu+1)-2 \nu}}\right)
$$

for all $n \in \mathbb{N}$.

Remark 2. Note that the rate exponent in (26) $\frac{2 \nu}{p(\nu+1)-2 \nu}=\frac{2 \nu}{\nu+1}\left(p-\frac{2 \nu}{\nu+1}\right)^{-1}$, always lies in the interval $\left[0, \frac{1}{p-1}\right]$, since $\frac{2 \nu}{\nu+1} \in[0,1]$.

Moreover, note that Theorem 1 provides a results on rates only, but no convergence result without variational inequality. This corresponds to the situation from [1] in a Hilbert space setting.

Proof. First of all, for $x_{n}^{\delta} \in \mathcal{B}_{\rho}^{D}\left(x^{\dagger}\right)$, (13) allows us to estimate as follows (see also (16)) in case $c_{1} \in[0,1)$ :

$$
\begin{aligned}
& \left\|F\left(x_{n}^{\delta}\right)-F\left(x^{\dagger}\right)-A\left(x_{n}^{\delta}-x^{\dagger}\right)\right\| \\
& \leq K\left\|A\left(x_{n}^{\delta}-x^{\dagger}\right)\right\|^{c_{1}} D_{q}^{x_{0}}\left(x^{\dagger}, x_{n}^{\delta}\right)^{c_{2}} \\
& \leq C\left(c_{1}\right) K\left(\left\|A\left(x_{n}^{\delta}-x^{\dagger}\right)\right\|+D_{q}^{x_{0}}\left(x^{\dagger}, x_{n}^{\delta}\right)^{\frac{c_{2}}{1-c_{1}}}\right),
\end{aligned}
$$

where we have used the abbreviation $A=F^{\prime}\left(x^{\dagger}\right)$ and the elementary estimate

$$
a^{1-\lambda} b^{\lambda} \leq C(\lambda)(a+b) \text { with } C(\lambda)=\lambda^{\lambda}(1-\lambda)^{1-\lambda} \text { for } a, b \geq 0, \lambda \in(0,1),
$$

and therewith, by the second triangle inequality,

$$
\left\|A\left(x_{n}^{\delta}-x^{\dagger}\right)\right\| \leq \frac{1}{1-C\left(c_{1}\right) K}\left(\left\|F\left(x_{n}^{\delta}\right)-F\left(x^{\dagger}\right)\right\|+C\left(c_{1}\right) K D_{q}^{x_{0}}\left(x^{\dagger}, x_{n}^{\delta}\right)^{\frac{c_{2}}{1-c_{1}}}\right)
$$

as well as analogously

$$
\begin{aligned}
& \left\|F\left(x_{n}^{\delta}\right)-F\left(x^{\dagger}\right)-A_{n}\left(x_{n}^{\delta}-x^{\dagger}\right)\right\| \\
& \leq 2 C\left(c_{1}\right) K\left(\left\|A\left(x_{n}^{\delta}-x^{\dagger}\right)\right\|+D_{q}^{x_{0}}\left(x^{\dagger}, x_{n}^{\delta}\right)^{\frac{c_{2}}{1-c_{1}}}\right) \\
& \leq \frac{2 C\left(c_{1}\right) K}{1-C\left(c_{1}\right) K}\left(\left\|F\left(x_{n}^{\delta}\right)-F\left(x^{\dagger}\right)\right\|+D_{q}^{x_{0}}\left(x^{\dagger}, x_{n}^{\delta}\right)^{\frac{c_{2}}{1-c_{1}}}\right) .
\end{aligned}
$$


For any $n \leq n_{*}$ according to (22), by (7) we have

$$
\begin{aligned}
& D_{q}^{x_{0}}\left(x^{\dagger}, x_{n+1}^{\delta}\right)-D_{q}^{x_{0}}\left(x^{\dagger}, x_{n}^{\delta}\right) \\
& =D_{q}^{x_{0}}\left(x_{n}^{\delta}, x_{n+1}^{\delta}\right)+\left\langle J_{q}^{X}\left(x_{n}^{\delta}-x_{0}\right)-J_{q}^{X}\left(x_{n+1}^{\delta}-x_{0}\right), x^{\dagger}-x_{n}^{\delta}\right\rangle_{X^{*} \times X} \\
& =D_{q}^{x_{0}}\left(x_{n}^{\delta}, x_{n+1}^{\delta}\right)-\mu_{n}\left\langle j_{p}^{Y}\left(F\left(x_{n}^{\delta}\right)-y^{\delta}\right), A_{n}\left(x_{n}^{\delta}-x^{\dagger}\right)\right\rangle_{Y^{*} \times Y} \\
& \quad+\alpha_{n}\left\langle J_{q}^{X}\left(x^{\dagger}-x_{0}\right), x^{\dagger}-x_{n}^{\delta}\right\rangle_{X^{*} \times X} \\
& \quad-\alpha_{n}\left\langle J_{q}^{X}\left(x^{\dagger}-x_{0}\right)-J_{q}^{X}\left(x_{n}^{\delta}-x_{0}\right), x^{\dagger}-x_{n}^{\delta}\right\rangle_{X * \times X}
\end{aligned}
$$

where the terms on the right hand side can be estimated as follows.

By (6) and (8) we have

$$
\begin{aligned}
& D_{q}^{x_{0}}\left(x_{n}^{\delta}, x_{n+1}^{\delta}\right) \\
& \leq C_{q^{*}}\left\|J_{q}^{X}\left(x_{n+1}^{\delta}-x_{0}\right)-J_{q}^{X}\left(x_{n}^{\delta}-x_{0}\right)\right\|^{q^{*}} \\
& =C_{q^{*}}\left\|\alpha_{n} J_{q}^{X}\left(x_{n}^{\delta}-x_{0}\right)+\mu_{n} A_{n}^{*} j_{p}^{Y}\left(F\left(x_{n}^{\delta}\right)-y^{\delta}\right)\right\|^{q^{*}} \\
& \leq 2^{q^{*}-1} C_{q^{*}}\left(\alpha_{n}^{q^{*}}\left\|x_{n}^{\delta}-x_{0}\right\|^{q}+\mu_{n}^{q^{*}}\left\|A_{n}^{*} j_{p}^{Y}\left(F\left(x_{n}^{\delta}\right)-y^{\delta}\right)\right\|^{q^{*}}\right) \\
& \leq 2^{q^{*}-1} C_{q^{*}}\left(\alpha_{n}^{q^{*}}\left(2^{q-1}\left(\left\|x^{\dagger}-x_{0}\right\|^{q}+\frac{1}{c_{q}} D_{q}^{x_{0}}\left(x^{\dagger}, x_{n}^{\delta}\right)\right)+\mu_{n}^{q^{*}}\left\|A_{n}^{*} j_{p}^{Y}\left(F\left(x_{n}^{\delta}\right)-y^{\delta}\right)\right\|^{q^{*}}\right)\right.
\end{aligned}
$$

where we have used the triangle inequality in $X^{*}$ and $X$, the inequality

$$
(a+b)^{\lambda} \leq 2^{\lambda-1}\left(a^{\lambda}+b^{\lambda}\right) \text { for } a, b \geq 0, \lambda \geq 1,
$$

and (5).

For the second term on the right hand side of (31) we get, using (30), (28), (34),

$$
\begin{aligned}
&\left\langle j_{p}^{Y}\right.\left.\left(F\left(x_{n}^{\delta}\right)-y^{\delta}\right), A_{n}\left(x_{n}^{\delta}-x^{\dagger}\right)\right\rangle_{Y^{*} \times Y} \\
&=\left\langle j_{p}^{Y}\left(F\left(x_{n}^{\delta}\right)-y^{\delta}\right), F\left(x_{n}^{\delta}\right)-y^{\delta}\right\rangle_{Y^{*} \times Y} \\
&-\left\langle j_{p}^{Y}\left(F\left(x_{n}^{\delta}\right)-y^{\delta}\right), F\left(x_{n}^{\delta}\right)-y^{\delta}-A_{n}\left(x_{n}^{\delta}-x^{\dagger}\right)\right\rangle_{Y^{*} \times Y} \\
& \geq \frac{1-3 C\left(c_{1}\right) K}{1-C\left(c_{1}\right) K}\left\|F\left(x_{n}^{\delta}\right)-y^{\delta}\right\|^{p} \\
&-\left\|F\left(x_{n}^{\delta}\right)-y^{\delta}\right\|^{p-1}\left(\frac{2 C\left(c_{1}\right) K}{1-C\left(c_{1}\right) K} D_{q}^{x_{0}}\left(x^{\dagger}, x_{n}^{\delta}\right)^{\frac{c_{2}}{1-c_{1}}}+\frac{1+C\left(c_{1}\right) K}{1-C\left(c_{1}\right) K} \delta\right) \\
&= \frac{1-3 C\left(c_{1}\right) K}{1-C\left(c_{1}\right) K}\left\|F\left(x_{n}^{\delta}\right)-y^{\delta}\right\|^{p} \\
&-\left(\frac{1-3 C\left(c_{1}\right) K}{3 C\left(\frac{p-1}{p}\right)\left(1-C\left(c_{1}\right) K\right)}\left\|F\left(x_{n}^{\delta}\right)-y^{\delta}\right\|^{p}\right)^{\frac{p-1}{p}}\left(\frac{\left(3 C\left(\frac{p-1}{p}\right)\right)^{p-1}}{\left(1-C\left(c_{1}\right) K\right)}\right)^{\frac{1}{p}} \\
& \quad\left(2 C\left(c_{1}\right) K D_{q}^{x_{0}}\left(x^{\dagger}, x_{n}^{\delta}\right)^{\frac{c_{2}}{1-c_{1}}}+\left(1+C\left(c_{1}\right) K\right) \delta\right)
\end{aligned}
$$




$$
\begin{aligned}
\geq & \frac{1-3 C\left(c_{1}\right) K}{1-C\left(c_{1}\right) K}\left\|F\left(x_{n}^{\delta}\right)-y^{\delta}\right\|^{p}-C\left(\frac{p-1}{p}\right)\left\{\frac{1-3 C\left(c_{1}\right) K}{3 C\left(\frac{p-1}{p}\right)\left(1-C\left(c_{1}\right) K\right)}\left\|F\left(x_{n}^{\delta}\right)-y^{\delta}\right\|^{p}\right. \\
& \left.+\frac{\left(3 C\left(\frac{p-1}{p}\right)^{p-1}\right.}{\left(1-C\left(c_{1}\right) K\right)} 2^{p-1}\left(\left(2 C\left(c_{1}\right) K\right)^{p} D_{q}^{x_{0}}\left(x^{\dagger}, x_{n}^{\delta}\right)^{\frac{c_{2} p}{1-c_{1}}}+\left(1+C\left(c_{1}\right) K\right)^{p} \delta^{p}\right)\right\} .
\end{aligned}
$$

Using the variational inequality (11), (29), and

$$
(a+b)^{\lambda} \leq\left(a^{\lambda}+b^{\lambda}\right) \text { for } a, b \geq 0, \lambda \in[0,1],
$$

we get

$$
\begin{aligned}
& \left|\alpha_{n}\left\langle J_{q}^{X}\left(x^{\dagger}-x_{0}\right), x^{\dagger}-x_{n}^{\delta}\right\rangle_{X * \times X}\right| \\
& \leq \beta \alpha_{n} D_{q}^{x_{0}}\left(x^{\dagger}, x_{n}^{\delta}\right)^{\frac{1-\nu}{2}}\left\|F^{\prime}\left(x^{\dagger}\right)\left(x_{n}^{\delta}-x^{\dagger}\right)\right\|^{\nu} \\
& \leq \beta \alpha_{n} D_{q}^{x_{0}}\left(x^{\dagger}, x_{n}^{\delta}\right)^{\frac{1-\nu}{2}} \frac{1}{\left(1-C\left(c_{1}\right) K\right)^{\nu}}\left(\left\|F\left(x_{n}^{\delta}\right)-y^{\delta}\right\|+\delta+C\left(c_{1}\right) K D_{q}^{x_{0}}\left(x^{\dagger}, x_{n}^{\delta}\right)^{\frac{c_{2}}{1-c_{1}}}\right)^{\nu} \\
& \leq \beta \alpha_{n} D_{q}^{x_{0}}\left(x^{\dagger}, x_{n}^{\delta}\right)^{\frac{1-\nu}{2}} \epsilon^{-\nu}\left(\epsilon \frac{1}{\left(1-C\left(c_{1}\right) K\right)^{\nu}}\left(\left\|F\left(x_{n}^{\delta}\right)-y^{\delta}\right\|+\delta\right)\right)^{\nu} \\
& +\beta \alpha_{n}\left(\frac{C\left(c_{1}\right) K}{\left(1-C\left(c_{1}\right) K\right)}\right)^{\nu} D_{q}^{x_{0}}\left(x^{\dagger}, x_{n}^{\delta}\right)^{\frac{1-\nu}{2}+\frac{\nu c_{2}}{1-c_{1}}} \\
& \leq C\left(\frac{\nu}{p}\right)\left\{\left(\beta \alpha_{n} D_{q}^{x_{0}}\left(x^{\dagger}, x_{n}^{\delta}\right)^{\frac{1-\nu}{2}} \epsilon^{-\nu}\right)^{\frac{p}{p-\nu}}+\left(\epsilon \frac{1}{\left(1-C\left(c_{1}\right) K\right)^{\nu}}\left(\left\|F\left(x_{n}^{\delta}\right)-y^{\delta}\right\|+\delta\right)\right)^{p}\right\} \\
& +\beta \alpha_{n}\left(\frac{C\left(c_{1}\right) K}{\left(1-C\left(c_{1}\right) K\right)}\right)^{\nu} D_{q}^{x_{0}}\left(x^{\dagger}, x_{n}^{\delta}\right)^{\frac{1-\nu}{2}+\frac{\nu c_{2}}{1-c_{1}}} \\
& =C\left(\frac{\nu}{p}\right)\left\{\left(\beta \epsilon^{-\nu}\right)^{\frac{p}{p-\nu}}\left(3 C\left(\frac{\nu}{p}\right) C\left(\frac{p(1-\nu)}{2(p-\nu)}\right)\right)^{\frac{p(1-\nu)}{2(p-\nu)}} \alpha_{n}^{\frac{p(1+\nu)}{2(p-\nu)}}\left(\frac{\alpha_{n} D_{q}^{x_{0}}\left(x^{\dagger}, x_{n}^{\delta}\right)}{3 C\left(\frac{\nu}{p}\right) C\left(\frac{p(1-\nu)}{2(p-\nu)}\right)}\right)^{\frac{p(1-\nu)}{2(p-\nu)}}\right\} \\
& \left.+\left(\epsilon \frac{1}{\left(1-C\left(c_{1}\right) K\right)^{\nu}}\left(\left\|F\left(x_{n}^{\delta}\right)-y^{\delta}\right\|+\delta\right)\right)^{p}\right\} \\
& +\beta \alpha_{n}\left(\frac{C\left(c_{1}\right) K}{\left(1-C\left(c_{1}\right) K\right)}\right)^{\nu} D_{q}^{x_{0}}\left(x^{\dagger}, x_{n}^{\delta}\right) \frac{1-\nu-c_{1}+\nu c_{1}+2 \nu c_{2}}{2\left(1-c_{1}\right)} \\
& \leq C\left(\frac{\nu}{p}\right)\left\{C ( \frac { p ( 1 - \nu ) } { 2 ( p - \nu ) } ) \left[\left(\beta \epsilon^{-\nu}\left(3 C\left(\frac{\nu}{p}\right) C\left(\frac{p(1-\nu)}{2(p-\nu)}\right)\right)^{\frac{1-\nu}{2}}\right)^{\frac{2 p}{p(\nu+1)-2 \nu}} \alpha_{n}^{\frac{p(1+\nu)}{p(\nu+1)-2 \nu}}\right.\right. \\
& \left.+\left(\frac{\alpha_{n} D_{q}^{x_{0}}\left(x^{\dagger}, x_{n}^{\delta}\right)}{3 C\left(\frac{\nu}{p}\right) C\left(\frac{p(1-\nu)}{2(p-\nu)}\right)}\right)\right] \\
& \left.+\left(\epsilon \frac{1}{\left(1-C\left(c_{1}\right) K\right)^{\nu}}\left(\left\|F\left(x_{n}^{\delta}\right)-y^{\delta}\right\|+\delta\right)\right)^{p}\right\} \\
& +\frac{1}{3} \alpha_{n} D_{q}^{x_{0}}\left(x^{\dagger}, x_{n}^{\delta}\right)
\end{aligned}
$$


where we have used (28) two times and $\epsilon>0$ will be chosen as a sufficiently small number below. Moreover, by (15), the exponent $\frac{1-\nu-c_{1}+\nu c_{1}+2 \nu c_{2}}{2\left(1-c_{1}\right)}=1+$ $\frac{1+\nu}{2\left(1-c_{1}\right)}\left(c_{1}+\frac{2 \nu}{\nu+1} c_{2}-1\right)$ is larger or equal to one and $\beta$ is sufficiently small so that $\beta\left(\frac{C\left(c_{1}\right) K}{\left(1-C\left(c_{1}\right) K\right)}\right)^{\nu} \rho^{\frac{1-\nu-c_{1}+\nu c_{1}+2 \nu c_{2}}{2\left(1-c_{1}\right)}-1}<\frac{1}{3}$.

Finally, we have that

$$
\begin{aligned}
\left\langle J_{q}^{X}\left(x^{\dagger}-x_{0}\right)-J_{q}^{X}\left(x_{n}^{\delta}-x_{0}\right), x^{\dagger}-x_{n}^{\delta}\right\rangle_{X^{*} \times X} & =D_{q}^{x_{0}}\left(x^{\dagger}, x_{n}^{\delta}\right)+D_{q}^{x_{0}}\left(x_{n}^{\delta}, x^{\dagger}\right) \\
& \geq D_{q}^{x_{0}}\left(x^{\dagger}, x_{n}^{\delta}\right)
\end{aligned}
$$

Inserting estimates (32)-(38) with $\epsilon=2^{p-1} \mu_{n}^{1 / p}\left(\frac{1-3 C\left(c_{1}\right) K}{3\left(1-C\left(c_{1}\right) K\right)}\right)^{1 / p} \frac{\left(1-C\left(c_{1}\right) K\right)^{\nu}}{C\left(\frac{\nu}{p}\right)}$ into (31) and using boundedness away from zero of $\mu_{n}$ and the abbreviations

$$
\begin{aligned}
\mathrm{d}_{n}= & D_{q}^{x_{0}}\left(x^{\dagger}, x_{n}^{\delta}\right)^{1 / 2} \\
C_{0}= & 6^{p-1} C\left(\frac{p-1}{p}\right)^{p} \frac{\left(2 C\left(c_{1}\right) K\right)^{p}}{\left(1-C\left(c_{1}\right) K\right)} \\
C_{1}= & 2^{q^{*}+q-2} \frac{C_{q^{*}}}{c_{q}} \\
C_{2}= & C\left(\frac{\nu}{p}\right) C\left(\frac{p(1-\nu)}{2(p-\nu)}\right)\left(\beta \epsilon^{-\nu}\left(3 C\left(\frac{\nu}{p}\right) C\left(\frac{p(1-\nu)}{2(p-\nu)}\right)^{\frac{1-\nu}{2}}\right)^{\frac{2 p}{p(\nu+1)-2 \nu}}\right. \\
C_{3}= & 2^{q^{*}+q-2} C_{q^{*}}\left\|x^{\dagger}-x_{0}\right\|^{q} \\
C_{4}= & 2^{p-1} C\left(\frac{\nu}{p}\right) \bar{\epsilon} \frac{1}{\left(1-C\left(c_{1}\right) K\right)^{\nu}}+6^{p-1} C\left(\frac{p-1}{p}\right)^{p} \frac{\left(1+C\left(c_{1}\right) K\right)^{p}}{1-C\left(c_{1}\right) K} \\
& \quad \underline{\epsilon}^{p}=2^{p-1} \underline{\mu}^{1 / p}\left(\frac{1-3 C\left(c_{1}\right) K}{3\left(1-C\left(c_{1}\right) K\right)}\right)^{1 / p} \frac{\left(1-C\left(c_{1}\right) K\right)^{\nu}}{C\left(\frac{\nu}{p}\right)} \\
& \quad \bar{\epsilon}=2^{p-1} \bar{\mu}^{1 / p}\left(\frac{1-3 C\left(c_{1}\right) K}{3\left(1-C\left(c_{1}\right) K\right)}\right)^{1 / p} \frac{\left(1-C\left(c_{1}\right) K\right)^{\nu}}{C\left(\frac{\nu}{p}\right)}
\end{aligned}
$$

we obtain

$$
\begin{aligned}
& \mathrm{d}_{n+1}^{2} \leq C_{0} \mathrm{~d}_{n}^{\frac{2 c_{2} p}{1-c_{1}}}+\left(1-\frac{1}{3} \alpha_{n}+C_{1} \alpha_{n}^{q^{*}}\right) \mathrm{d}_{n}^{2}+C_{2} \alpha_{n}^{\frac{p(1+\nu)}{p(\nu+1)-2 \nu}}+C_{3} \alpha_{n}^{q^{*}}+C_{4} \delta^{p} \\
& -\left(\mu_{n} \frac{1-3 C\left(c_{1}\right) K}{3\left(1-C\left(c_{1}\right) K\right)}\left\|F\left(x_{n}^{\delta}\right)-y^{\delta}\right\|^{p}-2^{q^{*}+q-2} C_{q^{*}} \mu_{n}^{q^{*}}\left\|A_{n}^{*} j_{p}^{Y}\left(F\left(x_{n}^{\delta}\right)-y^{\delta}\right)\right\|^{q^{*}}\right) .
\end{aligned}
$$

Here the last term is nonpositive due to the choice (18) of $\mu_{n}$, so that we arrive at

$$
\mathrm{d}_{n+1}^{2} \leq C_{0} \mathrm{~d}_{n}^{\frac{2 c_{2} p}{1-c_{1}}}+\left(1-\frac{1}{3} \alpha_{n}+C_{1} \alpha_{n}^{q^{*}}\right) \mathrm{d}_{n}^{2}+\underbrace{\left(C_{2}+C_{3}+C_{4} \tau^{-p}\right)}_{=: C_{5}} \alpha_{n}^{\frac{p(1+\nu)}{p(\nu+1)-2 \nu}}
$$

where we have used (24) and the stopping rule (22). Denoting

$$
\gamma_{n}:=\frac{\mathrm{d}_{n}^{2}}{\alpha_{n}^{\frac{2 \nu}{p(\nu+1)-2 \nu}}}
$$


we get the following recursion

$\gamma_{n+1} \leq C_{0}\left(\frac{\alpha_{n}}{\alpha_{n+1}}\right)^{\theta} \alpha_{n}^{\theta \omega} \gamma_{n}^{\omega}+\left(\frac{\alpha_{n}}{\alpha_{n+1}}\right)^{\theta}\left(1-\frac{1}{3} \alpha_{n}+C_{1} \alpha_{n}^{q^{*}}\right) \gamma_{n}+C_{5}\left(\frac{\alpha_{n}}{\alpha_{n+1}}\right)^{\theta} \alpha_{n}$

with

$$
\theta=\frac{2 \nu}{p(\nu+1)-2 \nu} \quad \omega=\frac{c_{2} p}{1-c_{1}}
$$

where

$$
\omega \geq 1
$$

by (14) and

$$
\theta \omega=\frac{p}{p-\frac{2 \nu}{\nu+1}} \frac{c_{2} \frac{2 \nu}{\nu+1}}{1-c_{1}} \geq 1
$$

due to assumption (15). Hence as sufficient conditions for uniform boundedness of $\left\{\gamma_{n}\right\}_{n \leq n_{*}}$ by $\bar{\gamma}$ and for $x_{n+1}^{\delta} \in \mathcal{B}_{\rho}^{D}\left(x^{\dagger}\right)$ we get

$$
\begin{gathered}
\bar{\gamma} \leq \rho^{2} \\
C_{0} \alpha_{n}^{\theta \omega-1} \bar{\gamma}^{\omega}-\left\{\left(\frac{\alpha_{n+1}}{\alpha_{n}}\right)^{\theta}+\frac{1}{3} \alpha_{n}-1-C_{1} \alpha_{n}^{q^{*}}\right\} \alpha_{n}^{-1} \bar{\gamma}+C_{5} \leq 0,
\end{gathered}
$$

where by $q^{*}>1$, (15) the factors $C_{0} \alpha_{n}^{\theta \omega-1}, C_{1} \alpha_{n}^{q^{*}-1}$ and $C_{5}$ can be made small for small $\alpha_{\max }, \beta,\left\|x^{\dagger}-x_{0}\right\|$ and large $\tau$. We use this fact to achieve

$$
C_{0} \alpha_{n}^{\theta \omega-1} \rho^{\omega-1}+C_{1} \alpha_{n}^{q^{*}-1} \leq \tilde{c}<c
$$

with $\tilde{c}$ independent of $n$, which together with (23) yields sufficiency of

$$
\frac{C_{5}}{c-\tilde{c}} \leq \bar{\gamma} \leq \rho^{2}
$$

for (41), (42), which for any (even small) prescribed $\rho$ is indeed enabled by possibly decreasing $\beta,\left\|x^{\dagger}-x_{0}\right\|, \tau^{-1}$, and therewith $C_{5}$.

In case $c_{1}=1$, estimates (29), (30) simplify to

$$
\left\|A\left(x_{n}^{\delta}-x^{\dagger}\right)\right\| \leq \frac{1}{1-\rho^{2 c_{2}} K}\left\|F\left(x_{n}^{\delta}\right)-F\left(x^{\dagger}\right)\right\|
$$

and

$$
\left\|F\left(x_{n}^{\delta}\right)-F\left(x^{\dagger}\right)-A_{n}\left(x_{n}^{\delta}-x^{\dagger}\right)\right\| \leq \frac{2 \rho^{2 c_{2}} K}{1-\rho^{2 c_{2}} K}\left\|F\left(x_{n}^{\delta}\right)-F\left(x^{\dagger}\right)\right\| .
$$

Therewith, the terms containing $D_{q}^{x_{0}}\left(x^{\dagger}, x_{n}^{\delta}\right)^{\frac{c_{2}}{1-c_{1}}}$ are removed and $C\left(c_{1}\right)$ is replaced by $\rho^{2 c_{2}}$ in (32)-(38), so that we end up with a recursion of the form (40) (with $C_{0}$ replace by zero) as before. Hence the remainder of the proof of uniform boundedness of $\gamma_{n}$ can be done in the same way as in case $c_{1}<1$. 
In case $\delta=0$, i.e., $n_{*}=\infty$, uniform boundedness of $\left\{\gamma_{n}\right\}_{n \in \mathbb{N}}$ implies (26). For $\delta>0$ we get (25) by using (22) in

$$
D_{q}^{x_{0}}\left(x^{\dagger}, x_{n_{*}}\right)=\gamma_{n_{*}} \alpha_{n_{*}}^{\frac{2 \nu}{p(\nu+1)-2 \nu}} \leq \bar{\gamma} \alpha_{n_{*}}^{\frac{2 \nu}{p(\nu+1)-2 \nu}} \leq \bar{\gamma}(\tau \delta)^{\frac{2 \nu}{\nu+1}}
$$

Remark 3. In view of estimate (39), an optimal choice of $\alpha_{n}$ would be one that minimizes the right hand side. At least in the special case that the same power of $\alpha_{n}$ appears in the last two terms, i.e., $\frac{p(1+\nu)}{p(\nu+1)-2 \nu}=q^{*}$, elementary calculus yields

$$
\left(\alpha_{n}^{o p t}\right)^{\frac{2 \nu}{p(\nu+1)-2 \nu}}=\frac{D_{q}^{x_{0}}\left(x^{\dagger}, x_{n}^{\delta}\right)}{3 q^{*}\left(C_{1} D_{q}^{x_{0}}\left(x^{\dagger}, x_{n}^{\delta}\right)+C_{5}\right)},
$$

which shows that the obtained relation $D_{q}^{x_{0}}\left(x^{\dagger}, x_{n}^{\delta}\right) \sim \alpha_{n}^{\frac{2 \nu}{p(\nu+1)-2 \nu}}$ is indeed reasonable and probably even optimal.

\section{References}

1. Bakushinsky, A.B., Kokurin, M.Y.: Iterative methods for approximate solution of inverse problems. Springer, Dordrecht (2004)

2. Burger, M., Osher, S.: Convergence rates of convex variational regularization. Inverse Problems 20(5), 1411-1421 (2004)

3. Clason, C., Jin, B.: A semi-smooth Newton method for nonlinear parameter identification problems with impulsive noise. SIAM J. Imaging Sci. 5, 505-538 (2012)

4. Hein, T., Hofmann, B.: Approximate source conditions for nonlinear ill-posed problems - chances and limitations. Inverse Problems 25, 035003 (16pp) (2009)

5. Hein, T., Kazimierski, K.: Accelerated Landweber iteration in Banach spaces. Inverse Problems 26, 055002 (17pp) (2010)

6. Hofmann, B., Kaltenbacher, B., Pöschl, C., Scherzer, O.: A convergence rates result for Tikhonov regularization in Banach spaces with non-smooth operators. Inverse Problems 23(3), 987-1010 (2007)

7. Kaltenbacher, B., Hofmann, B.: Convergence rates for the iteratively regularized Gauss-Newton method in Banach spaces. Inverse Problems 26, 035007 (21pp) (2010)

8. Kaltenbacher, B., Neubauer, A., Scherzer, O.: Iterative Regularization Methods for Nonlinear Ill-posed Problems. de Gruyter (2007)

9. Kaltenbacher, B., Schöpfer, F., Schuster, T.: Convergence of some iterative methods for the regularization of nonlinear ill-posed problems in Banach spaces. Inverse Problems 25, 065003 (2009), doi: 10.1088/0266-5611/25/6/065003

10. Neubauer, A., Hein, T., Hofmann, B., Kindermann, S., Tautenhahn, U.: Improved and extended results for enhanced convergence rates of Tikhonov regularization in Banach spaces. Appl. Anal. 89(11), 1729-1743 (2010)

11. Scherzer, O.: A modified Landweber iteration for solving parameter estimation problems. Appl. Math. Optim. 38, 45-68 (1998)

12. Schöpfer, F., Louis, A.K., Schuster, T.: Nonlinear iterative methods for linear illposed problems in Banach spaces. Inverse Problems 22(1), 311-329 (2006)

13. Schuster, T., Kaltenbacher, B., Hofmann, B., Kazimierski, K.: Regularization Methods in Banach Spaces. de Gruyter (2007)

14. Hein, T., Kazimierski, K.S.: Modified Landweber iteration in Banach spaces convergence and convergence rates. Numerical Functional Analysis and Optimization 31(10), 1158-1189 (2010) 